\title{
Airborne observation of the Hayabusa sample return capsule re-entry
}

Jay H. Grinstead

NASA Ames Research Center

Moffett Field, CA 94035

Peter M. Jenniskens

The SETI Institute

Mountain View, CA 94041

Alan M. Cassell

ERC Incorporated

Moffett Field, CA 94035

Jim Albers

The SETI Institute

Mountain View, CA 94041

Michael Winter

University Affiliated Research Center/University of California, Santa Cruz Moffett Field, CA 94035

The Japan Aerospace Exploration Agency (JAXA) recently completed their Hayabusa asteroid exploration mission. Launched in 2003, Hayabusa made contact with, and retrieved a sample from, the near-Earth asteroid Itokawa in 2005. The sample return capsule (SRC) re-entered over the Woomera Test Range (WTR) in southern Australia on June 13, 2010, at approximately 11:21 pm local time (09:51 UTC). The SRC re-entry velocity was $12.2 \mathrm{~km} / \mathrm{s}$, making it the second-fastest Earth return velocity behind $\mathrm{NASA}^{1} \mathrm{~s}$ Stardust sample return capsule re-entry in 2006.

From a space technology development perspective, Hayabusa's re-entry functioned as a rare flight experiment of an entry vehicle and its thermal protection system. In collaboration with the SETI Institute, NASA deployed its DC-8 airborne laboratory and a team of international researchers to Australia to observe the re-entry of the SRC. The use of an airborne platform enables observation above most clouds and weather and greatly diminishes atmospheric absorption of the optical signals. The DC-8's flight path was engineered and flown to provide a view of the spacecraft that bracketed the heat pulse to the capsule. A suite of imaging instruments on board the DC-8 successfully recorded the luminous portion of the re-entry event. For approximately 70 seconds, the spectroscopic and radiometric instruments acquired images and spectra of the capsule, its wake, and destructive re-entry of the spacecraft bus. Figure 1 shows a perspective view of the WTR, the SRC re-entry trajectory, and the flight path of the DC- 8 .

The SRC was jettisoned from the spacecraft bus approximately 3 hours prior to entry interface. Due to thruster failures on the spacecraft, it could not be diverted from the entry path and followed the trajectory of the SRC, where it burned up in the atmosphere between approximately 100 and $50 \mathrm{~km}$ altitude. Fortuitously, the separation distance 
between the spacecraft and SRC was sufficient to clearly resolve the SRC from the debris field of the burning spacecraft. Figure 2 shows a frame from a high-definition television camera on board the aircraft and denotes the locations of the SRC and spacecraft bus debris.

Most instruments had the capability to spectrally resolve the emission of the SRC and spacecraft debris fragments. The spectral range covered by the instruments spanned from the near ultraviolet (approximately $300 \mathrm{~nm}$ ) to the short wave infrared (approximately $1700 \mathrm{~nm}$ ). The instruments were calibrated before and after the observation flight.

Reference standard irradiance source lamps were used for calibration to absolute spectral radiance. Atomic line source lamps were used for wavelength calibration. Atmospheric absorption will be corrected for using extinction calculations based on an atmosphere model and range-to-target distances. Figure 3 shows a preliminary spectrum recorded simultaneously by four separate instrument platforms; signal diminution due to atmospheric absorption in the infrared by $\mathrm{H}_{2} \mathrm{O}$ and $\mathrm{O}_{2}$ has not been corrected. Groundbased observation teams from the US, Australia, and Japan also recorded the re-entry. The ground and airborne observation data have been used to reconstruct the as-flown trajectory of the SRC.

The Hayabusa observation campaign's objectives and methods were similar to that of the Stardust re-entry observation. However, unique technical and programmatic challenges were encountered arising from coordination and cooperation with JAXA and the Australian authorities. A brief summary of the Hayabusa mission, the airborne observation campaign, data, and analysis will be presented.

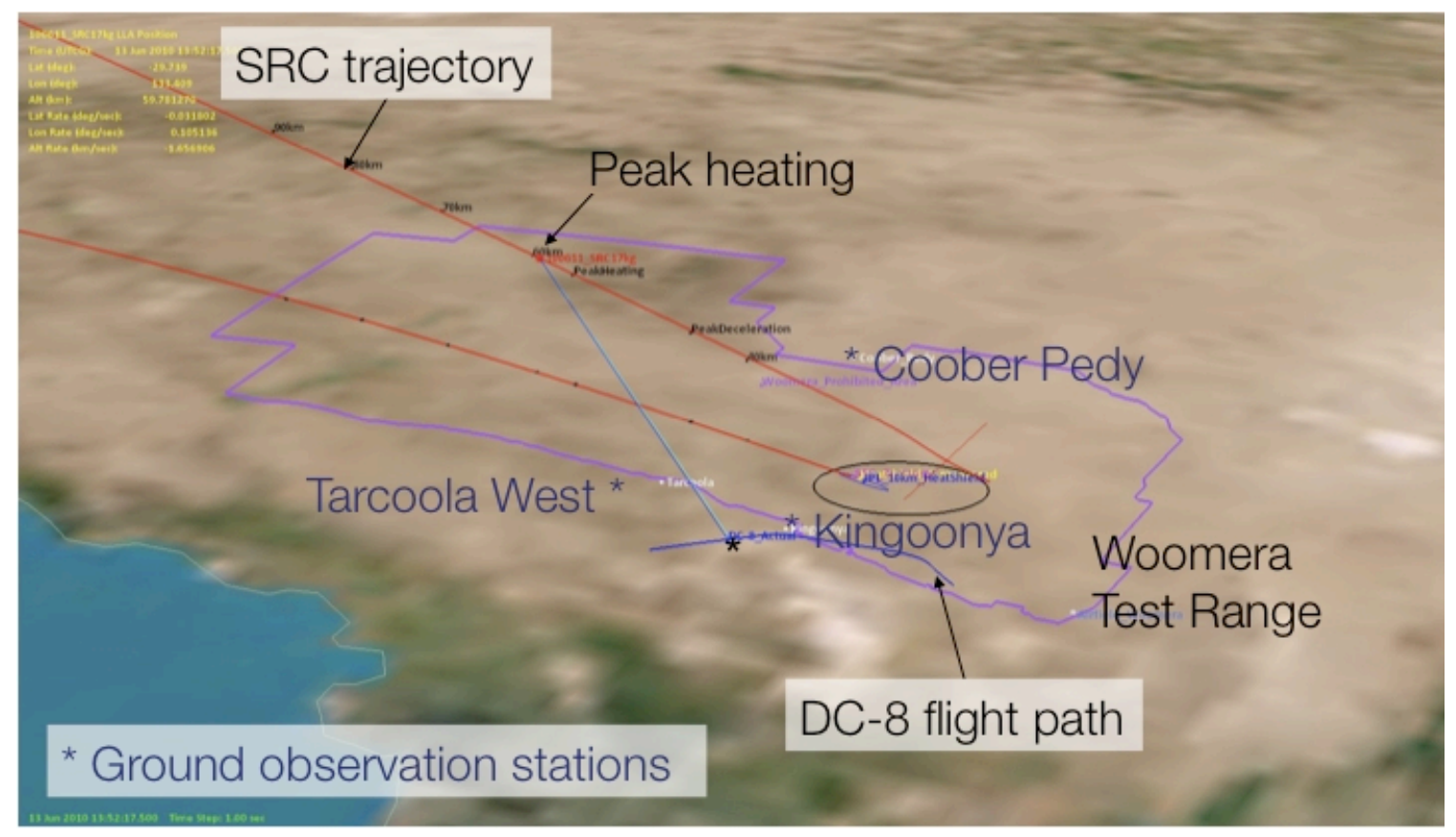

Figure 1. Perspective view of the Woomera Test Range in South Australia showing the re-entry trajectory of the Hayabusa SRC and the flight path of the DC-8 observation aircraft. Peak heating, predicted to occur at $58 \mathrm{~km}$ altitude, is noted. 


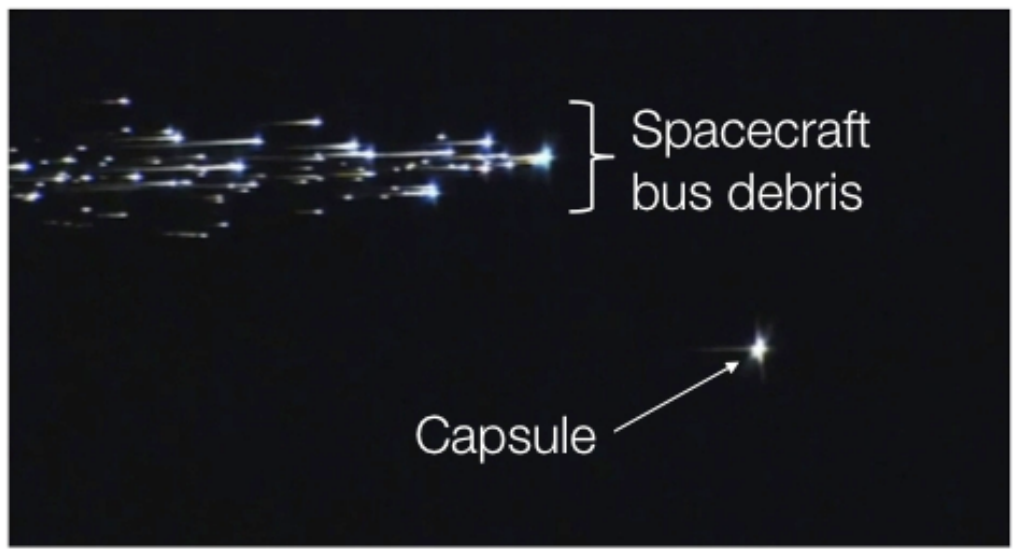

Figure 2. Single-frame image from a high-definition television camera aboard the DC-8 observation aircraft. The Hayabusa SRC is well separated from the burning debris of the spacecraft bus.

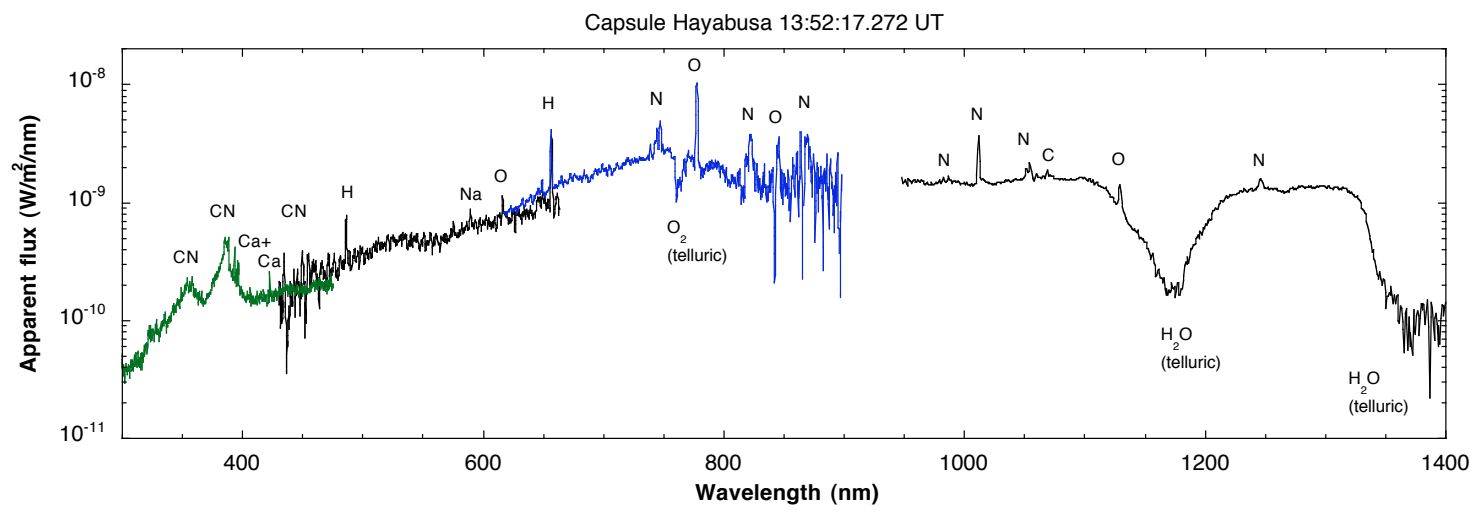

Figure 3. Composite spectrum of the SRC emission at one point in time as seen with four different instruments. Atomic and molecular emission features in the shock layer are noted. Absorption due to atmospheric $\mathrm{O}_{2}$ and $\mathrm{H}_{2} \mathrm{O}$ has not been corrected for. 